\title{
A Hybrid Technique Using Combinatorial Cyclic Difference Sets and Binomial Amplitude Tapering for Linear Sparse Array Antenna Design
}

\author{
Efri Sandi*, Fitri Yuli Zulkifli, and Eko Tjipto Rahardjo \\ Department of Electrical Engineering \\ Faculty of Engineering, Universitas Indonesia, Kampus Baru UI, Depok, Indonesia 16424 \\ *corresponding author, E-mail: efri.sandi@unj .ac.id
}

\begin{abstract}
Reducing system complexity and cost in synthesizing a sparse array antenna design is a challenging task for practical communication systems, such as radar systems and space communication. In this paper, a hybrid technique to synthesize a linear sparse array antenna design is described. This technique is developed using two methods. The first method is a combinatorial approach that applies cyclic difference sets (CDS) integers to significantly reduce the number of antenna elements. The approach and procedure used to apply the new CDS method to configure a linear sparse array, with significant reduction of the spatial antenna dimension, is described. The second method, applied to the array result of the first method, is amplitude tapering using a binomial array approach to reduce the sidelobes level (SLL). The simulation and measurement results of the sample sparse array design showed that the SLL was reduced in comparison to the sparse array design using only the combinatorial CDS method.
\end{abstract}

\section{Introduction}

Array antenna configurations are one of most useful antenna configurations in communication systems because they can provide a narrow beam width, variations in the beam pattern, directivity and high gain. High performance array antennas with very narrow beam widths and high directivity require large spatial apertures and a large number of antenna elements. These restrictions impact the antenna array sizing; thus, the communication system cost will be higher because of the many radio frequency (RF)/microwave components, filters and amplifiers that are required for each antenna element [1]. Therefore, reducing the number of antenna elements is a straightforward method to reduce the cost of a communication system.

In recent decades, antenna array designs have been developed with sparse element spacing characteristics. A sparse antenna array will achieve a desired radiation pattern with the minimum number of antenna elements, which is particularly useful in practical communication systems where the antenna weight and size are limited, such as in radar-phased antenna arrays, astronomy communications and satellite communications[1-2].

In the literature, various techniques have been proposed to configure sparse array antenna designs. Generally, there are five sparse array antenna design methods that have been published; they can be classified as deterministic and generic algorithm methods [3-9], stochastic and probabilistic methods [10-12], general polynomial factorizations [13-15], combinatorial methods [16-18] and mutual coupling effects [19-21]. The combinatorial method, using cyclic difference sets (CDS), is a suitable design for a sparse array antenna with high efficiency, a simple process and minimal computation time compared with other sparse array methods.

The primary issue of the combinatorial CDS method is the sidelobes level (SLL) performance. When applying the CDS integer to the sparse array configuration, the performance of the SLL is lower when compared to that of the full array configuration. In some applications, such as a radar system, the SLL performance will impact the scanning process and the radar measurement system. Therefore, SLL performance improvement is an important issue in sparse array antenna design.

The classical solution to improve array performance is amplitude tapering to define the excitation coefficients for each antenna element. Some methods have been developed by antenna designers for the amplitude tapering technique, such as the binomial, Dolph-Chebyshev, Taylor and cosine approaches. Each method has different characteristics and advantages for array performance and configuration.

In this study, we developed a combined approach using the combinatorial CDS method with amplitude tapering using a binomial array. We chose a binomial array because the distribution of coefficient is in staircase form, based on Pascal's triangle, which matches with our new approach using CDS configuration element distribution. After the introduction in section 1 , we describe the CDS integer approach and its application in the sparse array design in section 2. In section 3, we describe the hybrid technique, which applies amplitude tapering to the CDS configuration, and the proposed binomial array approach. In section 4, the simulation of a sample linear sparse array design to verify the proposed technique is presented, and the conclusion is presented in section 5 .

\section{New Combinatorial CDS Linier Sparse Array Design Approach}

Difference sets are branches of the mathematical combinatorial theory, which are suitable for the design of a 
sparse array antenna; this reduced a significant number of array elements [16-17]. By definition, a $(V, K, \Lambda)$ difference set is a set of $K$ unique integers based on $V$ integers from all populations and $\Lambda$ is possible unique differences appears sequence. In sparse array design, $K$ unique integers can be used as a series of array elements based on $V$ integers of full array element series.

From the set $D=\left\{d_{0}, d_{1}, \ldots, d_{K-1}\right\}$, with $0 \leq d i \leq(V-1)$, such that for any integer $1 \leq \alpha \leq(V-1)$,

where: $d_{i}-d_{j}=\alpha(\bmod V), i \neq j$

These three parameters $(V, K, \Lambda)$ are used to describe a difference set with only two independent parameters because there are $K(K-1)$ possible difference $\left(d_{i}-d_{j}\right)$ with $i$ not equal to $j$ and because each $(V-1)$ possible unique differences appears exactly $\Lambda$ times.

The three CDS parameters will have the following relation:

$$
K(K-1)=\Lambda(V-1)
$$

Example CDS parameters and integers are

$D 1\{1,3,4,5,9\}$; where $V=11, K=5, \Lambda=2$

D2 $\{1,2,4\}$; where $V=7, K=3, \Lambda=1$

D3 $\{3,6,7,12,14\}$; where $V=21, K=5, \Lambda=1$

$D 4\{0,1,2,4,5,10\}$; where $V=15, K=7, \Lambda=3$

$D 5\{1,4,5,6,7,9,11,16,17\}$; where $V=19, K=9, \Lambda=4$

The construction of a linear sparse array using CDS [16] is developed by using "one” and "zero" sequences following the CDS integers. From difference sets D, we may construct a sequence one and zero.

$$
A V=\{a i\} i=0,1,2, \ldots, V-1
$$

where $a_{j}=1$ if $\mathrm{j}$ is in $D$ and $a_{j}=0$ if $\mathrm{j}$ is not in $D$. For example, $D 1\{1,3,4,5,9\}$ gives rise to $A V=\{01011100010\}$. The linear sparse array configuration can be constructed by starting with an empty lattice of element locations spaced $\lambda / 2$ apart, placing an element at each location where a sequence has a "1" and skipping each location where the sequence has a "0" [16]. This approach can reduce the array by a significant number of elements from a full array configuration or minimize the array to approximately half of the number of elements $(K / V)$.

The challenge when using the CDS approach for sparse array design is reducing the high SLL as compared to a full array configuration [22]. In antenna array design, amplitude tapering is a classical solution to improve SLL performance by controlling amplitude excitation for each array element. The basic idea in amplitude tapering is the application of a normal distribution or a Gaussian distribution model to the power distribution. Following this principle, a new strategy for combinatorial CDS application to linear sparse array design is developed.

The new strategy for applying a CDS integer in the sparse array configuration is applied following the characteristic of the amplitude tapering distribution function, which is the primary contribution of the radiation pattern emanating from the center of the array distribution (Gaussian distribution model). Using this approach, the new design procedure is as follows:

1. Split the full array configuration into two sides from the center of the array.

2. Define the array configuration as a representative of the $V$ integer series for each side.

3. Apply the CDS $K$ integer series for the sparse array configuration on each side.

4. Reduce the spatial antenna dimension for each side of the array configuration.

For example, a 32-element linear array microstrip antenna with inter-element spacing of $\lambda / 2$ is reduced to a 16-element linear sparse array using our combinatorial CDS method. We choose a CDS $(15,7,3)$ for each side of the array configuration (16 elements per side). The sparse array element configuration following a CDS $K$-integer series places the element in the configuration of a $D$ set integer (0 1245810 ) element position, with one element in the center of the array for each side. The array configuration is shown in Fig. 1.

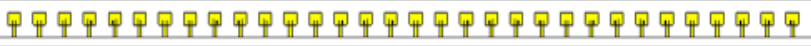
a. 32-element full-array antenna $(d=0.5 \lambda)$

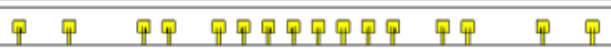

b. Reduced number of elements

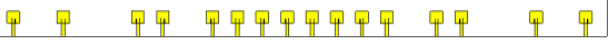

c. Reduced spatial antenna dimension

Figure 1: Design procedure of new CDS approach

\section{Hybrid Technique Applying Binomial Amplitude Tapering}

The proposed new configuration combinatorial CDS method for designing a linear sparse array antenna in the previous section is an effective method to reduce a significant number of elements, but this method still has the challenging issue of poor SLL performance. In the combinatorial CDS method, SLL performance was described using a relation between $K$ and $V$ [16, 22] as follows:

$$
\begin{aligned}
\text { PSL }= & 10 \log \left[\frac{1}{K}\left(1-\frac{K}{V}\right)\right] \\
& +10 \log [0.8488+1.128 \log V] d B
\end{aligned}
$$

Where PSL is peak SLL. The approximation is valid for $V>50$ and $K<V / 2$ [16, 23].

Using the approach in (4), the SLL performance in a combinatorial CDS is poor. In some applications, such as radar systems and satellite communication, the SLL performance is important for an accurate scanning result. To 
improve the SLL performance, an amplitude tapering solution is proposed. The procedure follows the new strategy combinatorial CDS configuration as described in section 2 and controls the excitation of each element by amplitude tapering.

Amplitude tapering is a type of non-uniform amplitude excitation for array elements. The most practical approaches and methods to determine amplitude tapering include a binomial array, a Dolph-Chebyshev array and a Taylor array. Each approach has advantages and disadvantages, depending on the desired design target and antenna array application requirement. Usually, a compromise must be made between the primary array antenna parameter, the half-power beam width (3-dB beam width), the directivity and a low SLL. As the SLL decreases, the half-power bandwidth decreases and typically, the directivity increases [24- 25].

To apply amplitude tapering to the new strategy CDS configuration, the binomial array can be applied directly, i.e., without a different excitation coefficient, unlike the DolphChebyshev and Taylor distribution models. The coefficient amplitude tapering methods for the Dolph-Chebyshev array and the Taylor array are similar. The coefficient tapering is distributed to all elements, with slight differences among them. The application significantly impacts array performance, especially after reducing the number of elements in the array distribution using CDS integers. The result is different when binomial array distribution, which follows Pascal's triangle, is applied; most of the distribution is in the center of the array. This model is similar to the proposed configuration of the CDS element position, which focuses on placing the array element in the center of the array. The coefficients for the edge distributions on both sides are small, especially for a large number of array elements, and can be neglected without any significant impact to array performance.

The binomial array was investigated and proposed by J.S Stone [24] to synthesize a pattern without sidelobes. Consider an array factor from two elements as follows:

$$
A F=1+e^{j \psi}
$$

Where $\psi=k d \cos \theta$

For a broadside array $(\beta=0)$ when inter-element spacing is less than half of a wavelength, the array factor has no sidelobes [24]. Continuing for three elements, the array factor produces the following:

$$
\begin{aligned}
3 \text { Elements } A F & =\left(1+e^{j \psi}\right)\left(1+e^{j \psi}\right) \\
& =\left(1+e^{j \psi}\right)^{2} \\
& =1+2 e^{j \psi}+e^{j \psi}
\end{aligned}
$$

This representation is the square of a two-element array factor without a sidelobe product and will also not have sidelobes. A similar approach is applied for four elements up to $N$ elements as follows:

$$
4 \text { elements } \begin{aligned}
A F & =\left(1+e^{j \psi}\right)^{2} \\
& =1+3 e^{j \psi}+3 e^{j \psi}+e^{j \psi}
\end{aligned}
$$

and for $N$ elements, the array factor will become $(1+$ $\left.e^{j \psi}\right)^{N-1}$ and

$$
N \text { elements } A F=\left(1+e^{j \psi}\right)^{N-1}
$$

Mathematically, the array factor shown in (8) represents a distribution of amplitude excitation for each element. Three elements have a ratio of 1:2:1 amplitude excitation and four elements have a ratio of 1:3:3:1. The excitation amplitude distribution can be easily obtained by expansion of the binomial in (8). Using Pascal's triangle, the distribution is as follows:

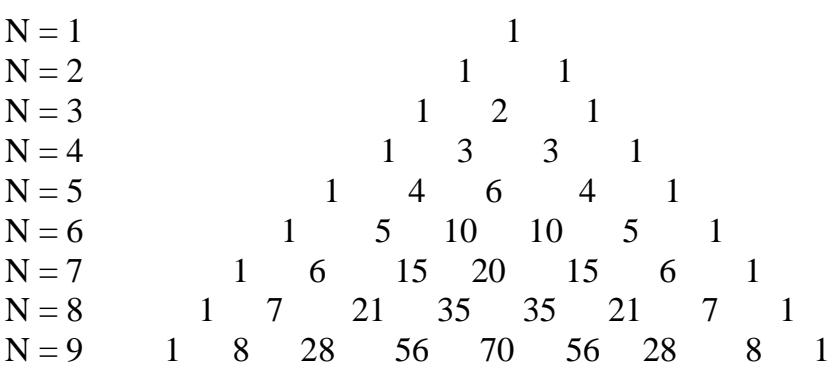

Based on the expansion of Pascal's triangle above, the coefficient for the edge distribution of both sides is small compared to the coefficient in the center distribution. This also occurs in the sparse array configuration based on the new CDS approach in which the eliminated element is placed on the edges of both sides. The new proposed CDS approach with binomial array amplitude tapering is therefore a hybrid technique linear sparse array antenna.

\section{Simulated and Measured Result}

To validate the proposed hybrid technique, as an example, a linear sparse array design for a 16-element linear full array is reduced to an 8-element linear sparse array using the procedure described in the previous section. In the simulation, a linear microstrip array antenna is used. It is designed at a frequency of $5 \mathrm{GHz}$ and uses a substrate material of Taconic TLY-5 $\left(\varepsilon_{\mathrm{r}}=2.2\right)$ with a thickness $=1.58$ in. Using Computer Simulation Technology (CST) microwave studio 2014 (https://www.cst.com) software simulation, we start by developing the 16-element full-array configuration as shown in Fig. 2. This configuration is the basis and parameter comparison for designing the linear sparse array antenna. The simulation and measurement results of s-parameter and radiation performance are shown in Fig. 3.

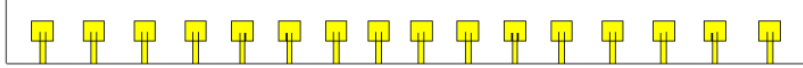

Figure 2: 16-element full-array antenna $(d=0.5 \lambda)$ 


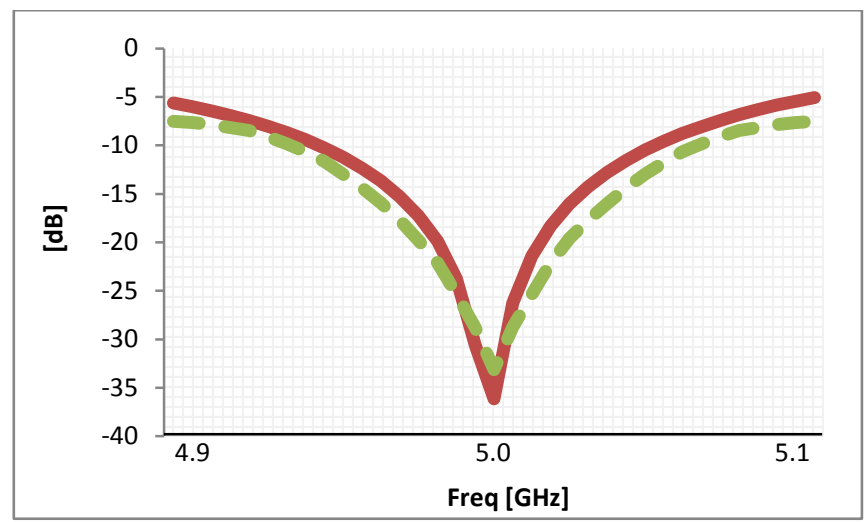

- Simulation a. $\mathrm{S}_{11}$

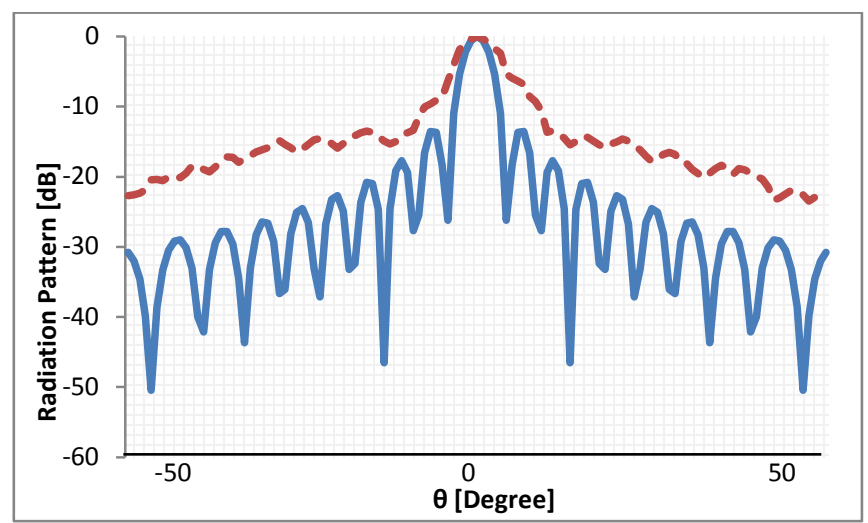

- Simulation --- Measurement b. 2D radiation pattern plot

Figure 3: Radiation performances of 16-element full-array antenna $(d=0.5 \lambda)$

The design result of the 16-element full-array antenna was a gain of $18.8 \mathrm{~dB}$ with a half-power beam width of $4.5^{\circ}$ and an SLL of $-13.6 \mathrm{~dB}$. The next step in designing a linear sparse array is splitting the configuration into two sides of eight elements each. CDS $(7,3,1)$ is then applied to this split configuration as shown in Fig. 4a.

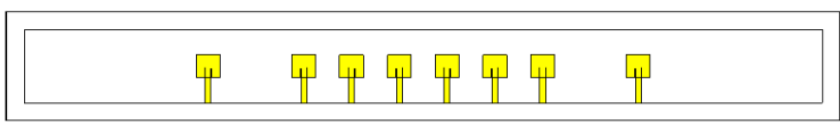

a. Reduced number of elements with $\operatorname{CDS}(7,3,1)$

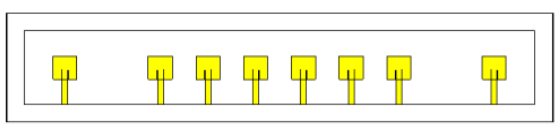

b. Reduced spatial antenna dimension

Figure 4: Design process of 8-element sparse array

In the next step of the sparse array design, the spatial dimension is reduced by decreasing the antenna length (substrate) at the edges of both sides, resulting in the final configuration as shown in Fig. 4b. The simulation and measurement results of the s-parameter and the radiation performance are shown in Fig. 5.

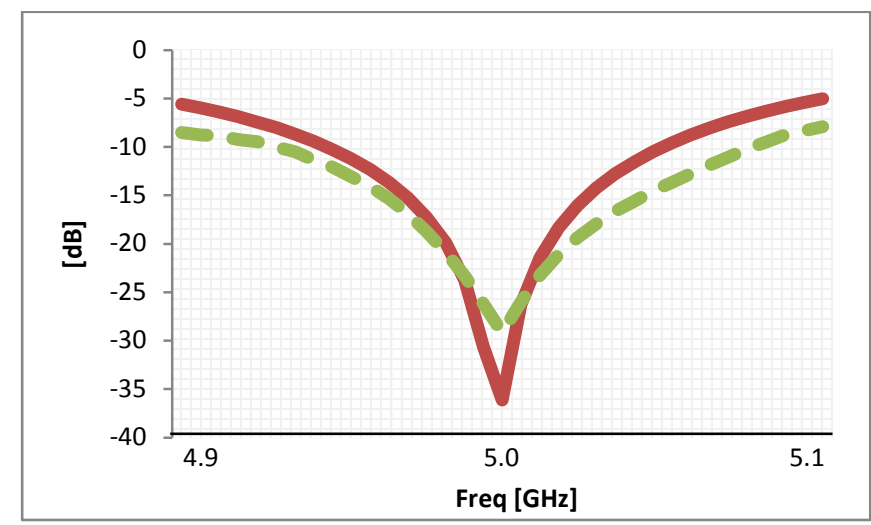

- Simulation --- Measurement

a. $\mathrm{S}_{11}$

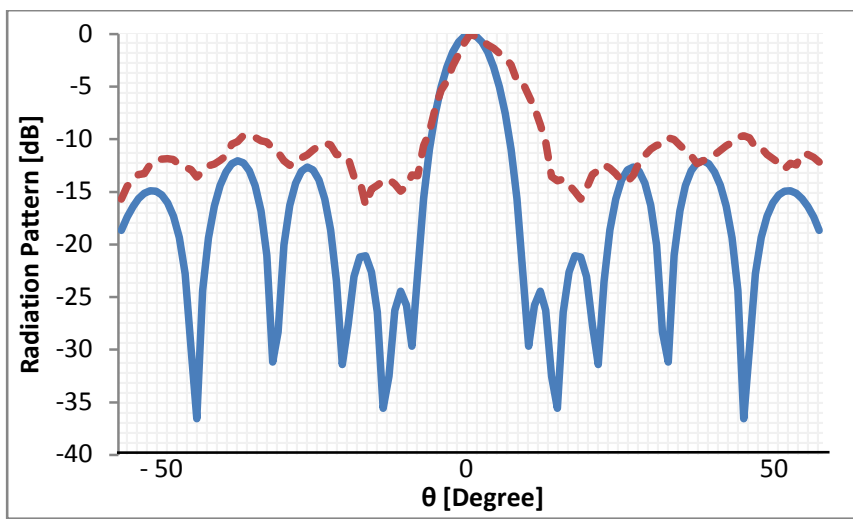

- Simulation --- Measurement

b. 2D radiation pattern plot

Figure 5: Radiation performances of 8-element sparse array by applying $\operatorname{CDS}(7,3,1)$

The simulation and measurement results show that the SLL of the radiation pattern linear sparse array using the CDS method is higher than that of the full-array configuration, as shown in Fig. 5. The SLL simulation is $12.0 \mathrm{~dB}$ with a $3 \mathrm{~dB}$ beam width of $7.8^{\circ}$ and a gain of 16.1 dB.

The last step of the design process is to apply the amplitude tapering using a binomial array. For $\mathrm{N}=16$, the expansion of Pascal's triangle is $1,15,106,462,1386,3038$, 5014, 6463, 6463, 5014, 3038, 1386, 462,106, 15, 1. The amplitude tapering coefficient for each element is shown in Table 1.

The coefficients and relative amplitude for each element listed in Table 1 is applied to the eight elements remaining after reduction by the CDS sparse configuration, i.e., element numbers 4, 6, 7, 8, 9, 10, 11 and 13. 
Table 1: Amplitude Tapering Coefficients.

\begin{tabular}{ccc}
\hline $\begin{array}{c}\text { Element } \\
\text { Number }\end{array}$ & Coefficient & $\begin{array}{c}\text { Amplitude } \\
\text { Attenuate } \\
(\mathrm{dB})\end{array}$ \\
\hline 1 & 0.0001 & -40.00 \\
2 & 0.0020 & -26.99 \\
3 & 0.0200 & -16.99 \\
4 & 0.0700 & -11.55 \\
5 & 0.2100 & -6.78 \\
6 & 0.4700 & -3.28 \\
7 & 0.7800 & -1.08 \\
8 & 1.0000 & 0.00 \\
9 & 1.0000 & 0.00 \\
10 & 0.7800 & -1.08 \\
11 & 0.4700 & -3.28 \\
12 & 0.2100 & -6.78 \\
13 & 0.0700 & -11.55 \\
14 & 0.0200 & -16.99 \\
15 & 0.0020 & -26.99 \\
16 & 0.0001 & -40.00 \\
\hline
\end{tabular}

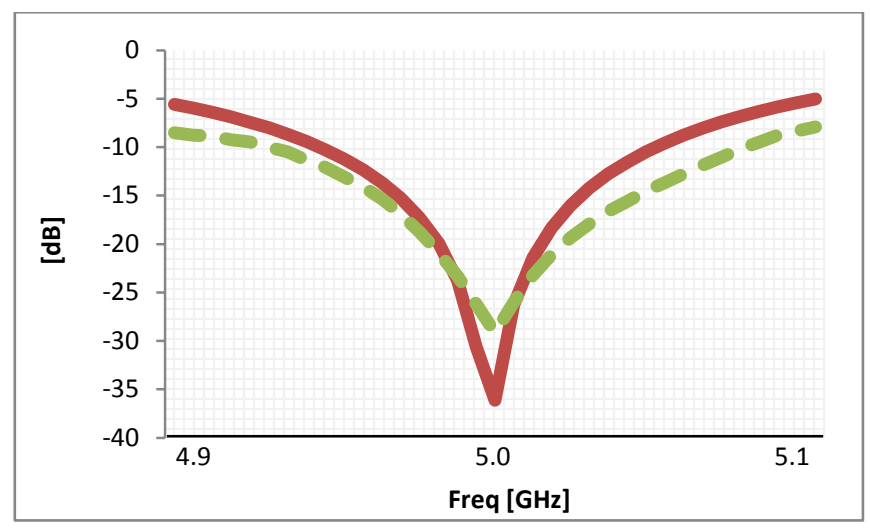

- Simulation --- Measurement a. $\mathrm{S}_{11}$

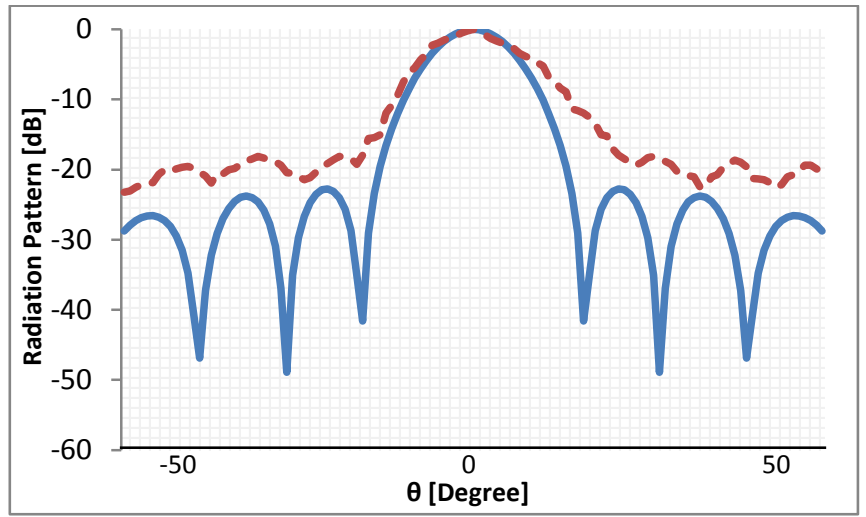

- Simulation

b. $2 \mathrm{D}$ radiation pattern

Figure 6: Radiation performances of 8-element hybrid technique sparse array antenna.
The simulation and measurement results of the proposed hybrid technique are shown in Fig. 6. The SLL simulation is -22.7 dB, a significant improvement over that of the combinatorial CDS linear sparse array. The implication of amplitude tapering is reduced directivity and a wider beam width, which are the compromises when the SLL is a priority in the antenna design requirements. The overall performance comparison is shown in Table 2.

Table 2: Performance Comparison of the hybrid technique for a 16-Element Array

\begin{tabular}{|c|c|c|c|c|c|}
\hline \multirow{3}{*}{ No } & \multirow{3}{*}{$\begin{array}{c}\text { Array } \\
\text { Configuration }\end{array}$} & \multirow{3}{*}{$\begin{array}{l}\text { Elements } \\
\text { \& } \\
\text { Aperture } \\
\text { Length }\end{array}$} & \multicolumn{3}{|c|}{$\begin{array}{c}\text { Radiation } \\
\text { Performances }\end{array}$} \\
\hline & & & & $3 \mathrm{~dB}$ & \\
\hline & & & $\begin{array}{l}\text { Gain } \\
(d B)\end{array}$ & $\begin{array}{c}\text { Beam } \\
\text { width } \\
\left({ }^{\circ}\right)\end{array}$ & $\begin{array}{l}\text { SLL } \\
\text { (dB) }\end{array}$ \\
\hline 1 & Full Array & $\begin{array}{c}16 \\
(16.83 \lambda)\end{array}$ & 18.8 & 4.5 & -13.6 \\
\hline 2 & $\begin{array}{l}\text { CDS Sparse } \\
\text { Array }\end{array}$ & $\begin{array}{c}8 \\
(10.78 \lambda)\end{array}$ & 16.1 & 7.8 & -12.0 \\
\hline 3 & $\begin{array}{l}\text { Hybrid } \\
\text { Technique } \\
\text { Sparse Array }\end{array}$ & $\begin{array}{c}8 \\
(10.78 \lambda)\end{array}$ & 14.5 & 13.1 & -22.7 \\
\hline
\end{tabular}

Using the same design procedure, the proposed hybrid technique is applied to the design of a 32- and a 64-element full array antenna to a sparse array configuration. The performance results are shown in Tables 3 and 4.

Table 3: Performance Comparison of the hybrid technique for a 32-Element Array

\begin{tabular}{|c|c|c|c|c|c|}
\hline \multirow{3}{*}{ No } & \multirow{3}{*}{$\begin{array}{c}\text { Array } \\
\text { Configuration }\end{array}$} & \multirow{3}{*}{$\begin{array}{l}\text { Elements } \\
\text { \& } \\
\text { Aperture } \\
\text { Length }\end{array}$} & \multicolumn{3}{|c|}{$\begin{array}{c}\text { Radiation } \\
\text { Performances } \\
\end{array}$} \\
\hline & & & & $3 \mathrm{~dB}$ & \\
\hline & & & $\begin{array}{l}\text { Gain } \\
(d B)\end{array}$ & $\begin{array}{c}\text { Beam } \\
\text { width } \\
\left(^{\circ}\right)\end{array}$ & $\begin{array}{l}\text { SLL } \\
\text { (dB) }\end{array}$ \\
\hline 1 & Full Array & $\begin{array}{c}32 \\
(32.94 \lambda)\end{array}$ & 21.8 & 2.2 & -13.6 \\
\hline 2 & $\begin{array}{l}\text { CDS Sparse } \\
\text { Array }\end{array}$ & $\begin{array}{c}16 \\
(24.89 \lambda)\end{array}$ & 19.2 & 3.2 & -11.9 \\
\hline 3 & $\begin{array}{l}\text { Hybrid } \\
\text { Technique } \\
\text { Sparse Array }\end{array}$ & $\begin{array}{c}16 \\
(24.89 \lambda)\end{array}$ & 16.1 & 8.8 & -22.1 \\
\hline
\end{tabular}

The overall performance comparison for larger number of elements is shown in Table 4. It is observed that the proposed hybrid technique has better efficiency for larger number of array (massively array) with significantly reduced number of array element and spatial antenna dimension. Furthermore, the simulation computation time speed proposed hybrid technique has better than CDS method and 
full array configuration. Using CST software simulation for 16-element array, the proposed technique has 424,116 mesh cells compared to 532,980 mesh cells for CDS method and 741,636 mesh cells for full array configuration. It is shown significant reduced computation time speed for time domain solver.

Table 4: Performance Comparison of the hybrid technique for a 64-Element Array

\begin{tabular}{|c|c|c|c|c|c|}
\hline \multirow[b]{2}{*}{ No } & \multirow[b]{2}{*}{$\begin{array}{c}\text { Array } \\
\text { Configuration }\end{array}$} & \multirow{2}{*}{$\begin{array}{l}\text { Elements } \\
\& \\
\text { Aperture } \\
\text { Length }\end{array}$} & \multicolumn{3}{|c|}{$\begin{array}{c}\text { Radiation } \\
\text { Performances }\end{array}$} \\
\hline & & & $\begin{array}{l}\text { Gain } \\
(d B)\end{array}$ & $\begin{array}{c}3 \mathrm{~dB} \\
\text { Beam } \\
\text { width } \\
\left(^{\circ}\right)\end{array}$ & $\begin{array}{l}\text { SLL } \\
(\mathrm{dB})\end{array}$ \\
\hline 1 & Full Array & $\begin{array}{c}64 \\
(65.17 \lambda)\end{array}$ & 24.8 & 0.6 & -23.2 \\
\hline 2 & $\begin{array}{l}\text { CDS Sparse } \\
\text { Array }\end{array}$ & $\begin{array}{c}32 \\
(63.16 \lambda)\end{array}$ & 22.3 & 0.72 & -10.9 \\
\hline 3 & $\begin{array}{l}\text { Hybrid } \\
\text { Technique } \\
\text { Sparse Array }\end{array}$ & $\begin{array}{c}16 \\
(26.9 \lambda)\end{array}$ & 17.3 & 6.6 & -21.1 \\
\hline
\end{tabular}

\section{Conclusions}

A new hybrid technique for the design of a linear sparse array antenna is described. The proposed method combines a new CDS approach with an amplitude tapering binomial array. The proposed new CDS configuration is a reduced spatial dimension array antenna that yields a significant reduction in the number of elements. The proposed hybrid technique for sparse array antenna design shows an improved SLL compared to both a combinatorial CDS linear sparse array antenna and a uniform amplitude fullarray configuration. The hybrid design has a significantly reduced number of elements, antenna dimensions and computation time speed.

\section{Acknowledgements}

The authors would like to acknowledge Hibah PUPT in the Ministry of Research, Technology and Higher Education, the Republic of Indonesia, for the research scheme under contract no. 0560/UN2.R12/HKP.05.00/2015.

\section{References}

[1] Coman, C. I., I. E. Lager, and L. P. Ligthart, "Design considerations in sparse array antennas," Proc. of 3rd European Radar Conference-EuRAD, Manchester, UK, Sept 2006.

[2] Robert, W., L. Xu, J. Li, P. Stoica, "Sparse antenna array design for MIMO Active Sensing Applications," IEEE Transaction on Antennas and Propagation, Vol. 59, No. 3, 846-858, 2011.
[3] Bucci, O. M., M. D’Urso, T.Isernia, P. Angeletti, and G. Toso, "Deterministic synthesis of uniform amplitude sparse arrays via new density taper techniques,” IEEE Transactions on Antennas and Propagation, Vol. 58, No. 6, 1949-1958, 2010.

[4] Caralelli, D., and M. C. Vigano, “A novel deterministic synthesis technique for constrained sparse array design problems," IEEE Transaction on Antennas and Propagation, Vol. 59, No. 11, 4085-4093, 2011.

[5] Bucci, O. M., T.Isernia, and A. F. Morabito, "An effective deterministic procedure for the synthesis of shaped beams by means of uniform-amplitude linear sparse arrays," IEEE Transactions on Antennas and Propagation, Vol. 61, No. 1, 169-175, 2013.

[6] Prisco, G., and M. D’Urso, “An effective approach for sparse array design with minimum number of sensors," Proc. of the 5th European on Antennas and Propagation (EUCAP); 2011.

[7] Yepes, L. F., D. H. Covarrubias, M. A. Alonso, and R. Ferrus, "Hybrid sparse linier synthesis applied to phased antenna arrays," IEEE Antennas and Wireless Propagation Letters, Vol. 13, 185-188, 2014.

[8] Roberts, W., L. Xu, J. Li, and P. Stoica, "Sparse antenna array design for MIMO active sensing applications," IEEE Transactions on Antennas and Propagation, Vol. 59, No. 3, 846-858, 2011.

[9] Mattew, B., and W. L. Hawes, "Location optimization of robust sparse antenna array with physical size contraint," IEEE Antennas and Wireless Propagation Letters, Vol. 11, 1303-1306, 2012.

[10] Minvielle, P., and Ph. Berisset, "Stochastic optimization of sparse array antenna,", European Conference on Antenna and Propagation, Barcelona 12-16 April, 2010.

[11] Minvielle, P., E. Tantar, A. Tantar, and P. Berisset, "Sparse antenna array optimization with the Crossentropy method,” IEEE Transactions on Antennas and Propagation, 2011, Vol. 59 No. 8, 2862-2871.

[12] Prisco, G., and M. D’Urso, “Maximally sparse array via Sequntial convex optimizations," IEEE Antennas and Wireless Propagationletters, Vol. 11, 192-195, 2012.

[13] Mitra, S. K., M. K. Tchobanau, and M. I. Bryukhanov, "A general method for designing sparse antenna array," Proc. of 2005 European Conference on Circuit Theory and Design, II/263-II266, 28 Aug.-2 Sept. 2005.

[14] Mitra, S. K., K. Mondal, M. K. Tchobanau, and G. J. Dolecek, "General polynomial factorization-based design od sparse periodic linier array," IEEE Transactions on Ultrasonic, Ferroelectrics, and Frequency Control, Vol. 57, No. 9, 1952-1966, 2010.

[15] Gustafsson, A., P. O. Fronlind, L. Pettersson,B. Carlegrim, and J.Svedin, "Measurement of sparse and compact phased array antenna architecture,” Proc. of 8th European Radar Conference (EuRAD), Manchester, UK 12-14 October, 412-415, 2011.

[16] Leeper, D. G., "Isophoric arrays-massively thinned phased arrays with well-controlled sidelobes," IEEE Transactions on Antennas and Propagation, Vol. 47, No. 12, 1825-1835, 1999. 
[17] Coman, C. I., I. E. Lager, and L. P. Ligthart, ”A deterministic solution to the problem of interleaving multiple sparse array antennas," European Microwave Conference, 4-6 October 2005.

[18]Dong, J., Q. Li, and W. Guo, “A combinatorial method for antenna array design in minimum redundancy MIMO radar," IEEE Antennas and Wireless Propagation Letters, 2009, Vol. 8, 1150-1153, 2009.

[19] Qu, S.W., M. Y. De-Jun He, Z. P. N. Xia, and C. H. Chan, "High-efficiency periodic sparse patch array based on mutual coupling," IEEE Antennas and Wireless Propagation Letters, Vol. 10, 1317-1320, 2011.

[20]Qu, S., C. H. Chan, M. Xia, and Z. Nie, "Highefficiency periodic sparse microstrip array based on mutual coupling," IEEE Transactions on Antennas and Propagation, Vol. 61, No. 4, 1963-1970, 2013.

[21] Pozar, D., “ Input impedance and mutual coupling of rectangular Microstrip antennas, “ IEEE Transactions on Antennas and Propagation, Vol. 30, No. 6, 1191-1196, 1982.

[22] Sandi, E., F. Y. Zulkifli, and E. T. Rahardjo, "Stretching strategy to improve radiation performances sparse array design based on combinatorial cyclic different sets approach," IEEE 4th Asia-Pacific Conference on Antennas and Propagat (APCAP), 161-162, 2015.

[23] Sandi, E., F.Y. Zulkifli, Basari and E.T. Rahardjo, “A Hybrid Technique Linier Sparse Array Antenna Design Approach," The 2015 International Symposium on Antennas and Propagation (ISAP2015), 744-746, 2015.

[24]Balanis, C. A. Antenna Theory-Analysis and Design. 3rd ed. New York: John Wiley \& Sons; 2005.

[25] Abed, A. T., "Study of radiation properties in Taylor distribution uniform space backfire antenna array," American Journal of Electromagnetics and Applications, Vol. 2, No. 3, 23-26, 2014. 